Aus dem Laboratorium der Universitäts-Frauenklinik der Königl. Charité zu Berlin.

\title{
Zur Kenntnis einiger Schädelanomalien der Neugeborenen; Schaltknochen und Defekte der Schädeldeckknochen ${ }^{1}$ ).
}

(I. Schaltknochen der hinteren Fontanelle, Spitzenknochen, Inkabein. II. Anomalien der vorderen Fontanelle; Anomalien der Form und Grösse, Verkïöcherung. III. Hypoplasie, Aplasie mid Defekte der Schädeldeckknochen. Eine besondere Form von Hydrocephalns.)

Van

\section{Prof. Dr. Robert Meyer.}

(Hierzu Tafel XVI-XX.)

M. H.! Gestatten Sie mir Ihnen Präparate und Abbildungen vorzulegen, wolche sie auf einige allerdings nicht unbekannto Anomalien des Schädels von Neugeborenen aufmerksam machen sollen. Vielleicht haben sie auch zum Teil geburtshilfliches Interesse, welches bisher nicht beachtet wurde, aber wohl in Zukunlt zur Geltung kommen mag.

Ich werde einige Befunde zu einem Demonstrationsvortrage vereinigen, welche auf verschiedenen Gebieten liegen und nicht den Anspruch einer fertigen Arbeit erheben, sondern zu weiterer $\Lambda$ rbeit anregen wollen.

\section{Schaltknochen der hinteren Fontanelle.}

Meine Aufmerksamkeit wurde auf die Schaltknochen der Fontanellen durch einen Zufall gerichtet; ein Neugeborenes fiel mir durch seinen relativ grossen Hinterkopf auf, wie Sie hier noch am Schädel sehen können (Fig. 3, Taf. XVI); bei Betastung der Nähte und Fontanellen konnte ich deutlich eine Transversalnaht an der Hinterhauptschuppe kurz unter der kleinen Fontanelle fühlen, so-

1) Dieser Vortrag wurde zu einer Demonstration abgekürzt am 28. Juli 11 in der Gesellschaft f. Geburtsh. u. Gyn. zu Berlin gehalten. 
dass es nicht möglich war mit einem Finger zu entscheiden, welches die Lambdanaht sei. Ich uberzeugte mich von der ungeheuer grossen Literatur, welche diese und verwandte Anomalien in anatomischer, entwicklungsgeschichtlicher, vergleichend anatomischer und anthropologischer Beleuchtung eingehend darstellt und war erstaunt darüber, dass sich in der geburtshilflichen Literatur keine klinischen Beobachtungen darüber finden.

Ich habe deshalb Neugeborene und ältere Feten von 5 Monaten aufwärts untersucht um zu erfahren, ob öfters solche grossen Schaltknochen vorkommen. Ehe ich ihnen meine Befunde genauer demonstriere, will ich nur kurz auf einige grössere Arbeiten hinweisen, von welchen aus die übrige Literatur erreichbar ist, eine Literatur, welche nach einem Ausspruch von Ranke eine ganze Bibliothek füllt. Von ihm stammt die letzte grössere Arbeit über diesen Gegenstand (Abh. d. math. phys. Cl. d. k. bayr. Akad. d. Wiss. 1900. Bd. 20. Abt. 2).

Dic Literatur über die Schaltknochen geht zurück bis auf das Jahr 1599, in welchem Ruini das sogenannte Os triquetrum beim Pferde als normale Bildung fand; bald wurde derselbe Knochen, welcher an Stelle des oberen Teiles der menschlichen Hinterhauptschuppe zwischen den Scheitelbeinen liegt und deshalb auch Os intraparietale (Geoffroy, Cuvier) genannt wird, auch bei anderen Säugern gefunden.

Man spricht auch von Os Goethianum (Fischer), weil Goethe den Intraparietalknochen bei der Maus fand. Beim Menschen sind ebenfalls seit langer Zeit verschiedene besonders abgegrenzte Knochenteile, sogenannte Schaltknochen als Anomalien der Hinterhauptschuppe bekannt, von denen Meckel den sogenannten Spitzenknochen als gleich bedeutend mit dem Os intraparietale der Tiere auffasste. Die grösseren Schaltknochen beim Menschen, welche die Schuppe betreffen, nennt man jetzt meistens Os Incae oder Inkabein nach Tschudi, weil er sie an Schädeln der Altperuaner besonders oft gefunden hatte. Es handelt sich hier um den grössten Teil der Hinterhauptoberschuppe, welche durch eine Transversalnaht von dem übrigen Hauptbein getrennt ist. In der normalen Entwicklung des Hinterhauptbeins besteht vorübergehend diese Transversalnaht, deren Reste Ihnen vielleicht unter dem Namen Sutura mendosa bekannt sind. Die Transversalnaht kann vollständig (Fig. 6) oder teilweise persistieren und je nachdem bleibt als selbständiger Knochen bestehen das Inkabein oder Teile eines solchen, 


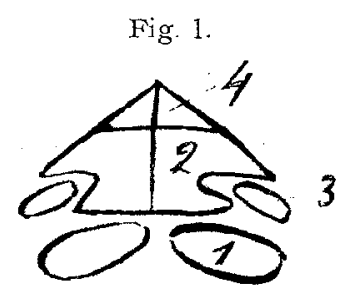

Fig. 1 nach Meckel.

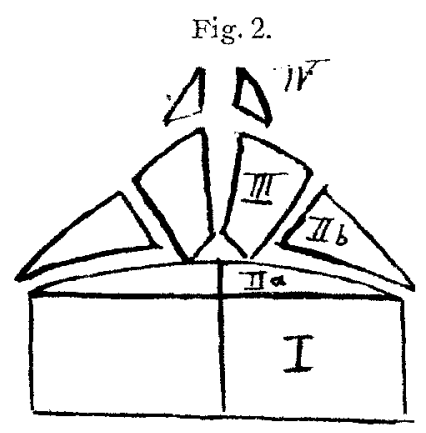

Fig. 2 nach Ranke.

Schema der Entwicklung der Hinterhauptschuppe aus einzelnen Knochenkernen. Fig. 1 nach Meckel, Fig. 2 nach Ranke.

Fig. 3.

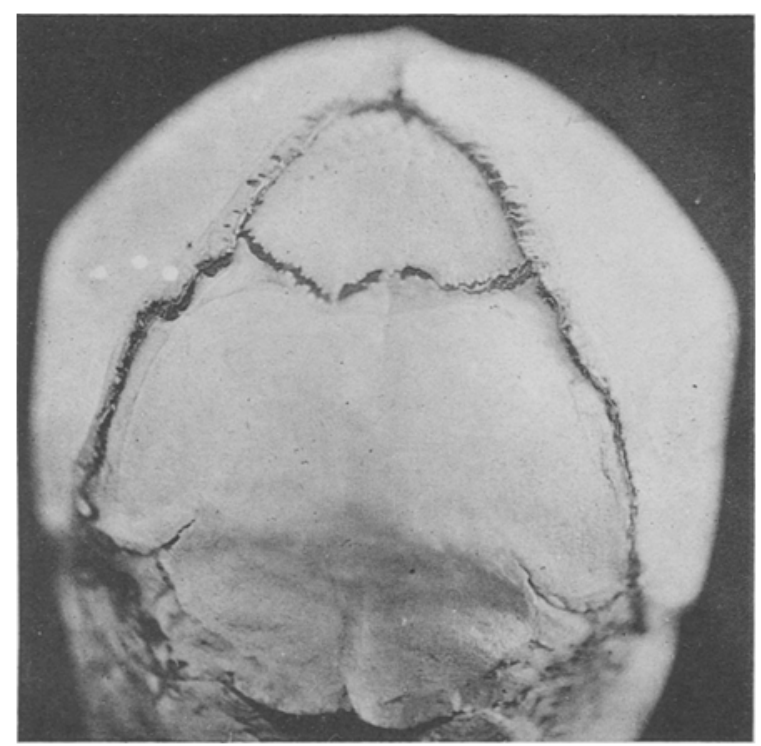

Neugeborenes, Dreieckiger Schaitknochen, sogenannter Spitzenknochen der hinteren Fontanelle. Palpierbarer Schaitknochen. 
ninkoide Bildungen" (Ranke). Ausser diesen Inkabeinen, welche seltener sind, unterscheidet man noch als häufigere Formen der Schaltknochen die schon erwähnten "Spitzenknochen" und die "Fontanellknochen". - Als Spitzenknochen bezeichnet man einen Schaltknochen, welcher meist dreieckige Form hat und aus der selbständigen oberen Spitzo der Hinterhauptschuppe besteht; der Spitzenknochen ist also nur ein Teil des Os intraparietale, welchem das Inkabein entspricht.

Virchow sieht alle Schaltknochen an der Spitze des Os occipitale als fonticulär an, welche eine mehr unregelmässige oder, wenn regelmässig, eine vierseitige mit einer medianen Spitze nach abwärts, zuweilen sogar sehr tief in die Schuppe eingreifende Gestalt besitzen, die dreicckigen dagegen mit einer einzigen Spitze gegen den Winkel der Lambdanaht gerichteten, und geradlinig gegen die Schuppe abgegrenzten Knochen als Teile der letzteren, als occipitale Bestandteile (Spitzenknochen).

Als Fontanellknochen werden meistens kleinere unregelmässige Schaltknochen der hinteren Fontanelle bezeichnet, welche jedoch in allen Uebergangsstufen zum Spitzenknochen vorkommen. Diese Spitzenknochen und Fontanellknochen sind es, von welchen beiden wir heute hauptsächlich sprechen wollen, ohne jedoch die Inkabeine ganz ausser acht lassen zu können, weil die Entstehungsgeschichte aller genannten Schaltknochen am Os occipitale von den Autoren eine gemeinsame und zwar sehr lebhafte Diskussion erfahren hat; an welcher sich Meckel, Virchow, Köllicker, Gegenbauer, Toldt, Graf von Spee, Welcker, Kollmann, O. Sehultze, Ranke und viele andere beteiligten (Literatur s. bei Ranke). Hervorgerufen wurden die Untersuchungen durch die merkwürdigen Verschiedenheiten der Schaltknochen, von welchen sich nur die Einteilungsprinzipien zweier grösserer Untersuchungsreilhen anführen will. Welcker unterscheidet Schädel mit vollständig erhaltener Transversalnaht der Hinterhauptschuppe und mit unvollständiger.

Bei ersteren ist das Os Incac simplex das häufigste, damn folgt das Os Incae tripartitum (13 p(t.), welches durch zwei seitliche nicht ganz sagittale Nähte in drei Teile zerlegt ist und schliesslich ein zweiteiliger Knochen, an welchem nur eine der beiden seitlichen Nähte vorhanden ist.

Bei der unvollkommenen Transversalnaht handelt es sich um sogenannte ,inkoide Bildungen", welche in allen denkbaren Variationen vorkommen unter Berücksichtigung der drei Knochen- 
Fig. 4.

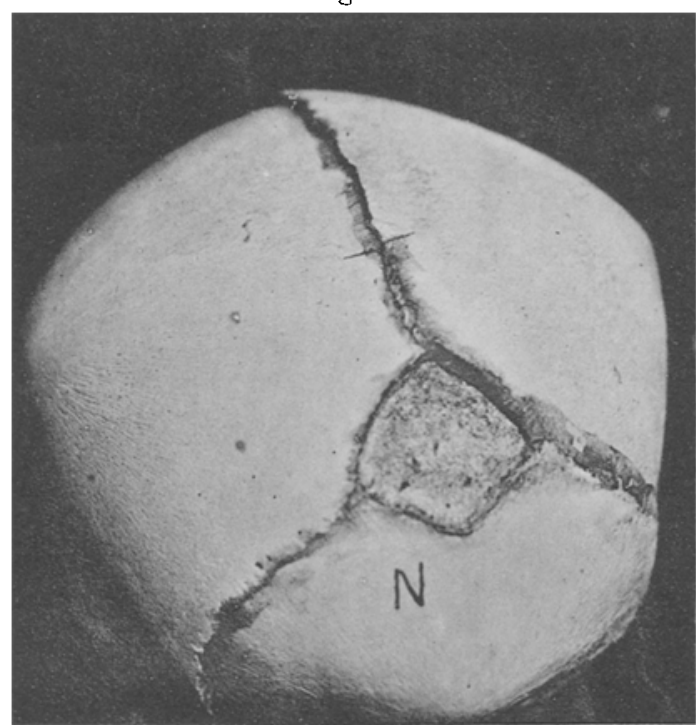

Neugeborenes. Viereckiger Spitzenknochen. Schaltknochen der hinteren Fontanelle unter Beeinträchtigung des Os occipitale.

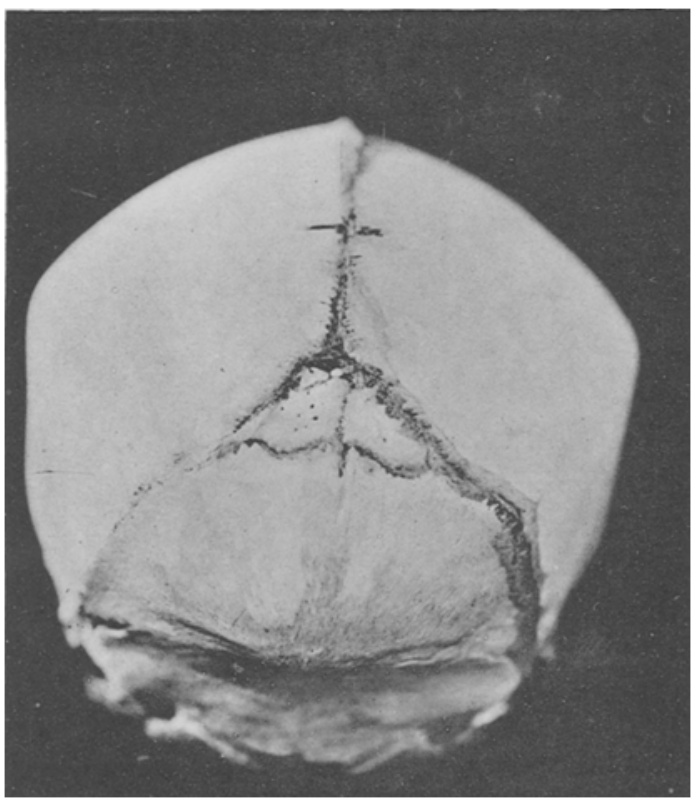

Fig. 5.

Neugeborenes. Zweiteiliger Spitzenknochen, doppelter Schaltknochen der hinteren Fontanelle. 
kernpaare. Nur die Mittelnaht bleibt kaum jemals erhalten bei Erwachsenen.

Welcker unterscheidet I. Ueberzählige Knochen, aus Knochenkernen oder Komplexen derselben, welche bei Tieren normal, beim Menschen ausnahmsweise selbständig bleiben. 1. Semifrontalbeine, 2. Os Incae, 3. Offenbleiben der Sutura squamosa pyramidalis. II. Separation frühester Kerne 1. der sogenannten Spitzenknochen aus dem 4. Kernpaare Meckels, 2. die Vereinigung des Spitzenknochens mit dem 2. Paare, 3. die den Seitendreiecken des Tripartium entsprechenden Stücke einzeln oder paarig. III. Unregelmässige Abspaltungen; Separation atypischer Kerne. IV. Fontanellknochen, Verknöcherungen in der Fontanellmembran, welche in der Lambdanaht nicht immer vom Spitzenknochen unterscheidbar sind. V. Nahtzwickelknochen oder Nahtdoppler. Die drei letzteren könnte man auch als atypische oder als accessorische Schaltknochen unter einer Rubrik zusammenfassen.

In Welckers Arbeit finden sie die Abbildungen der zahlreichen Varietäten nach seinem Einteilungsprinzip geordnet.

Ohne Kenntnis der Entwicklung sind die Anomalien des Hinterhauptbeines nicht verständlich und so hat schon Meckel versucht der Entwicklungsgeschichte gerecht zu werden. Meckel liess diese Hinterbauptsschuppe aus 8 Knochenpaaren hervorgehen, (s. Fig. 1 auf Taf. XVI) also aus 4 Paaren, während Virchow und Gegenbaur nur 2 Paare zugeben, aus deren einem Paar das Os intraparietale entstehe.

Welcker schliesst sich der Ansicht Meckels an. Nur unter Annahme von 3 Paar Knochenkernen seien die Varietäten des Os intraparietale verständlich und das 4 . Paar Knochenkerne bilde den Rest der Hinterhauptschuppe. Köllicker stellte fest, dass die Unterschuppe aus Knorpel, die Oberschuppe aus Bindegewebe entstehe, während man früher glaubte, die erste Anlage der Schuppe sei fibrös; R. Virchow fand, dass das Manubrium Kerkringii ebenfalls wie der übrige Teil der Unterschuppe aus Knorpel entstehe; seitdem war die prinzipielle Selbständigkeit der ganzen Oberschuppe als Hautknochen gegenüber dem knorplig präformierten "Primärknochen" (Unterschuppe und die ürigen Teile des Hinterhauptbeins) festgestellt.

Die umfangreiche Literatur der Entwicklung ist ron Graf v. Spee gesammelt; auch kann man die wichtigsten Literaturangaben bei Ranke einsehen. Es handelt sich hauptsächlich um 
Fig. 6.

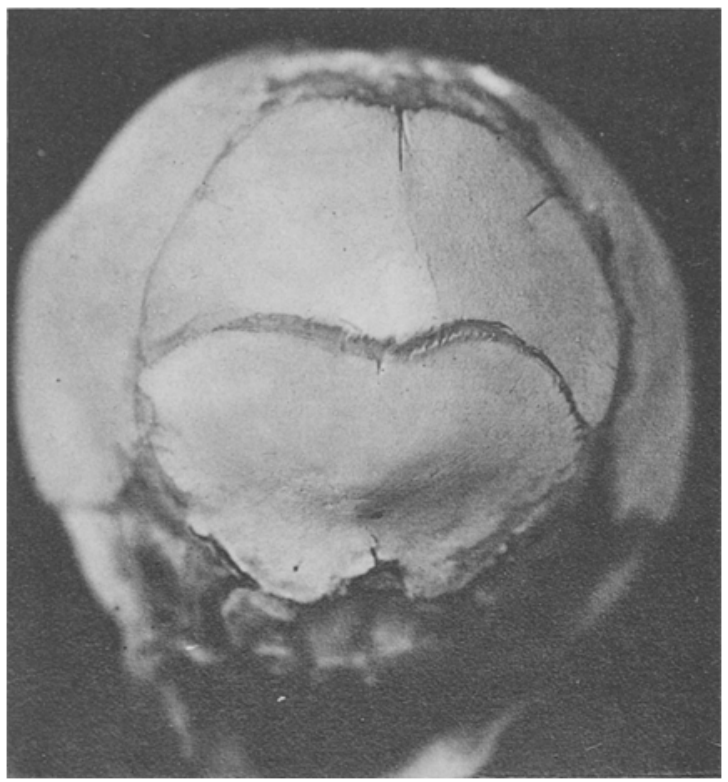

Neugeborenes. Transversale Nabt der Hinterhauptschuppe. Inkabein bei mässigem Grade von Hydrocephalus internus.

Fig. 7.

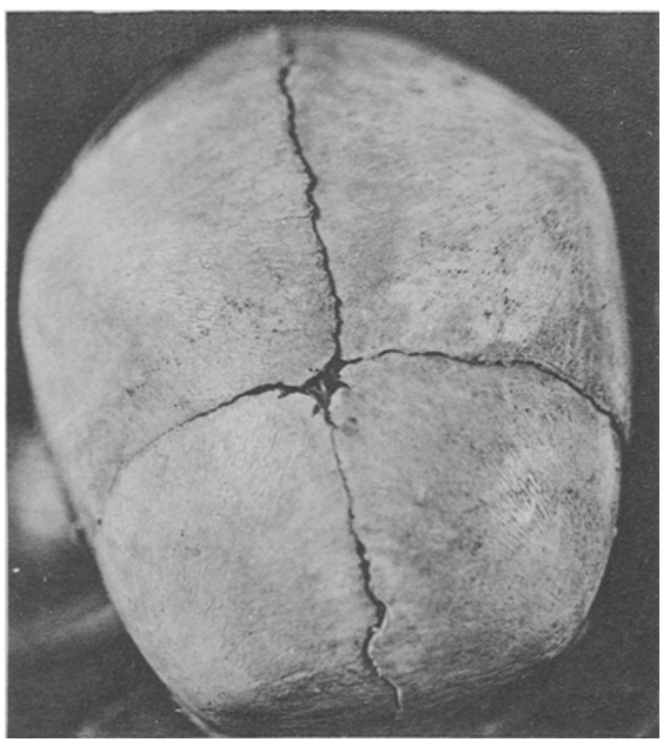

Neugeborenes. Vordere Fontanelle abnorm klein. 
die Zahl der Verknöcherungsprodukte und um die Stelle der Transversalnaht, welche zumejst als Grenze der Ober- und Unterschuppe angesehen wurde, während Bessel-Hagen behauptet, dass sie in der Oberschuppe selbst verläuft.

Die Meinungsverschiedenheiten waren bedingt durch die Schwierigkeit der Untersuchung: diese wurde erst durch. O. Schultze beseitigt durch Einführung eines neuen Hilfsmittels. An durchsichtig gemachten Feten konnte er die Knochenkerne demonstrieren und photographieren und nach dieser llethode gelang es auch Ranke, die Eatwicklung der Hinterhauptschuppe klarzulegen (s. Fig. 2 auf Taf. XVI).

Die Verknöcherung der Hinterhauptschuppe beginnt im Anfange des 3. Fetalmonates und ist am Ende desselben vollendet. Ranke fand 6 regelmässige Knochenkerne für die gesamte Hinterhauptschuppe, davon 2 für die Unterschuppe (I. Paar) und 4 für die Oberschuppe (II. und III. Paar). Das Kernpaar der Unterschuppe ist bei Ranke (s. Fig. 2) mit I bezeichnet, dann tritt ein Kernpaar II auf, welches erst neben der Mittellinie liegt und dann mit seinen oberen Partien lateralwärts auseinanderrückt. Nachdem die unteren Partien von II in der Mittellinie und mit der Unterschuppe verschmolzen sind, wird durch eine sekundär auftretende Transversalspalte, die sog. Sutura mendosa, der obere laterale Teil von II abgeschnitten, so dass der untere Teil II a der Unterschuppe angegliedert wird, während die oberen Teile IIb. rechts und links, zwischen denen medial ein Kernpaar III aufgetreten ist, sich mit diesen zur Oberschuppe vereinigen und verwachsen. Es verwachsen dann die Oberschuppe und Unterschuppe miteinander, doch bleibt der laterale Teil der Sutura mendosa noch bei den Neugeborenen sichtbar.' Wenn die Sutura mendosa gänzlich bestehen bleibt, so bildet der obere und grössere Teil der Oberschuppe das selbständige Os Incae. (Fig. 6, Taf. XVIII.)

Der Unterschied in der Anschauung Rankes von der Meckels. liegt also darin, dass er die Sutura mendosa, wie schon BesselHagen vor ihm nicht für primär hält, sondern für sekundär.

Das Entwicklungsschema Rankes unterscheidet sich meiner Meinung nach auch sonst wenig von dem Meckels, nur dass das. III. Paar Meckels bei Ranke als IIIb rangiert und umgekehrt Meckels II als Rankes III. Paar. Es handelt sich bei dieser Differenz nur um die Frage, welches Paar früher erscheint, nicht um verschiedene Topograghie, wie Ranke annimmt. 
Fig. 8.

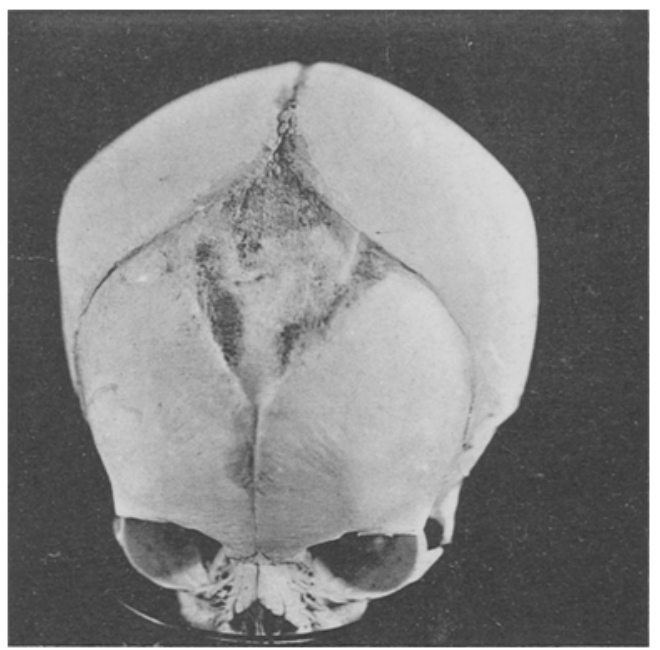

Fötus 8 mens. Vordere Fontanelle sehr gross, zum grossen Teil diffus verknöchert.

Fig. 9.

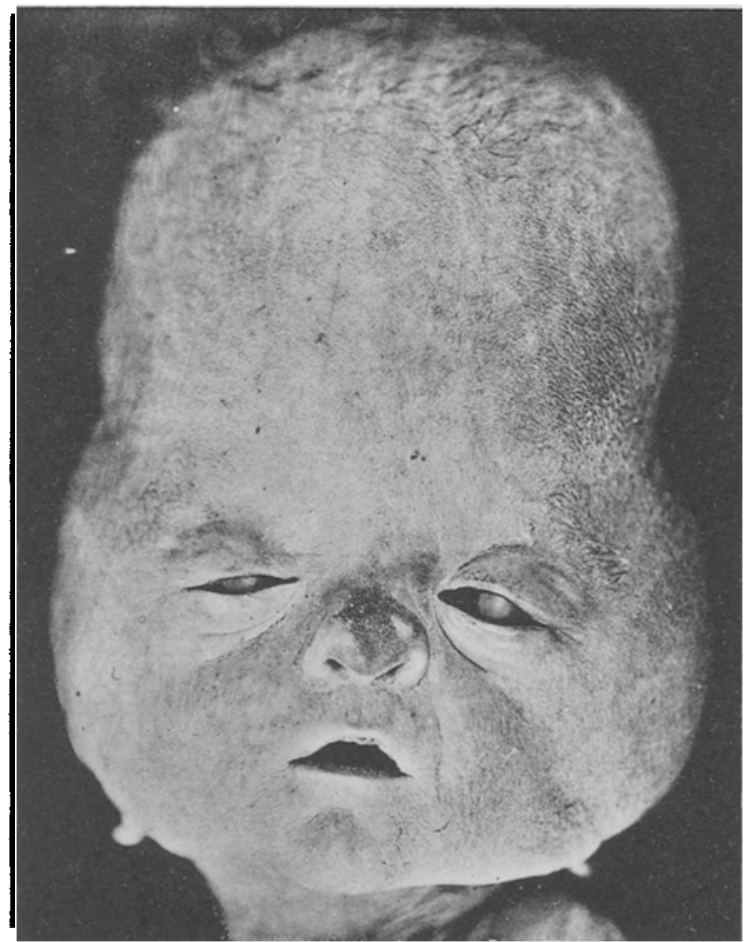

Hydrocephalus congenitus mit Schnürfurche der Stirn, Schläfengegend. Basaie Ohrstellung. 
Das 4. Kernpaar ist bei beiden Autoren gleich, nur mit dem Unterschiede, dass Meckel es für regulär hielt, während Ranke es nur atypisch fand; bei beiden entspricht das 4. Paar dem "Spitzenknochen".

Ton einer Atypie kann man aber nur bedingt sprechen, da die Spitzenknochen bei anderen Tieren regelmässig auftreten; mit den übrigen Schalt knochen der Nähte darf also das Spitzenbein nicht gleichgestellt werden.

Ranke dehnte seine Untersuchungen in grossem Massstabe auch auf die Schaltknochen aus und fand die Spitzenknochen stets erst nach dem 6. Monate, sie kommen bei Feten häufiger vor als bei Erwachsenen, 5 pCt. Bei älteren Feten unterscheidet Ranke Spitzenknochen und Fontanellknochen.

Die überzähligen Knochen teilt Ranke ein in accessorische und prinzipale; zu den ersteren gehören einerseits die Naht- und Fugenknochen, andererseits die Fontanellknochen, welche aus atypischen Knochenkernen entstehen. Deshalb rechnet Ranke auch die "Spitzenknochen" zu den accessorischen halbpathologischen Knochenbildungen; er stimmt also nicht mit Virchow überein, welcher, wie gesagt, die Spitzenknochen und Fontanellknochen auch der äusseren Form nach unterscheiden wollte.

In einer neueren Arbeit über die Entwicklung des Hinterhauptbeins kommt Bolk (1903) zu äbnlichen Resultaten wie Ranke.

Gestatten Sie mir nun meine Befunde von Schaltknochen der Fontanellen Ihnen vorzulegen.

\section{Typischer dreieckiger Spitzenknochen der hinteren Fontanelle vou besonderer Grösse. (Fig. 3, Taf. XVI.)}

Es ist das jener eingangs erwähnte Fall, den ich durch die Haut fühlen konnte. An dem länglichen Schädel fällt das niedrige Stirnbein im Vergleich mit dem grossen Hinterhaupte auf, besonders mit dem. Parietalknochen. Das Hinterhauptbein hat normale Grösse und Gestalt; die Schuppe ist normal gegen den Lateralteil abgesetzt, zwischen Oberschuppe und Unterschuppe ist die Sutura mendosa als lateraler Einschnitt noch sichtbar. Die Lambdanaht ist normal. Parietalbeine und und obere Spitze des Occipitalbeins stossen eng an einander. Die Hinterhauptschuppe ist durch eine breite gezackte transversale Naht in zwei Teile zerlegt; der obere Teil stellt den sogenannten Spitzenknochen dar und ist $2 \mathrm{~cm}$ in der Sagittallinie hoch, $3 \mathrm{~cm}$ breit, und annähernd dreieckig. Die Transversalnaht verläuft nicht ganz geradlinig sondern etwas uneben; sie ist am Schaltknochen fein gezackt, an der übrigen Schuppe nur wenig gezackt. Die Schuppe misst ron der abnormen 


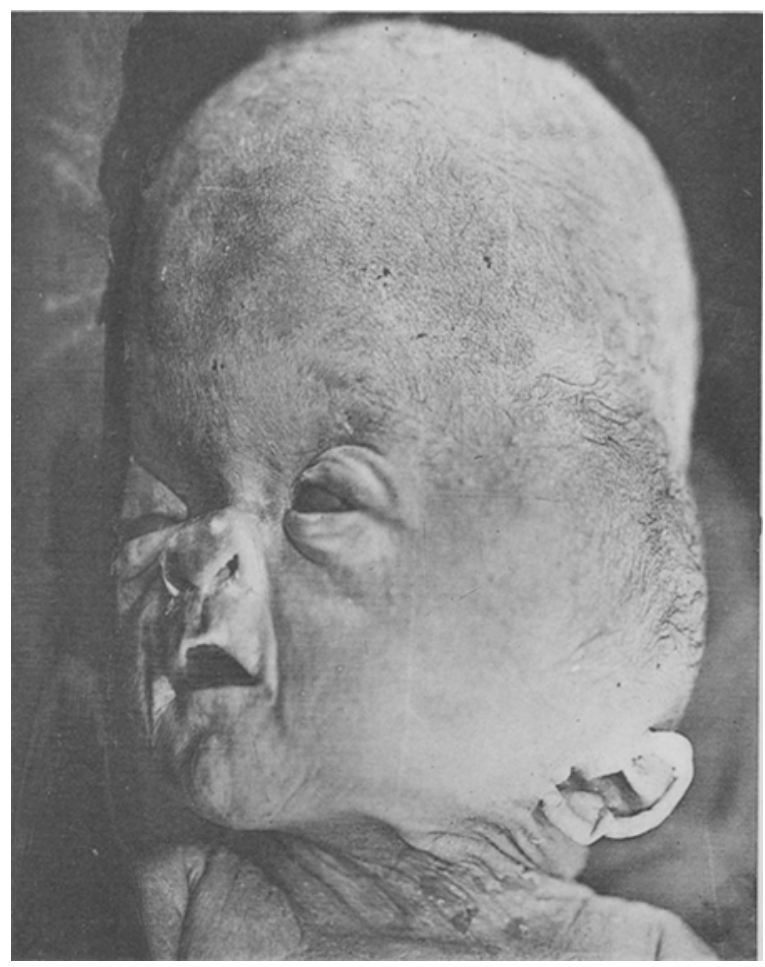

Hydrocephalus congenitus mit Schnürurche der Stirngegend Sch!äfe. Basale Ohrstellung.

Fig. 11 .

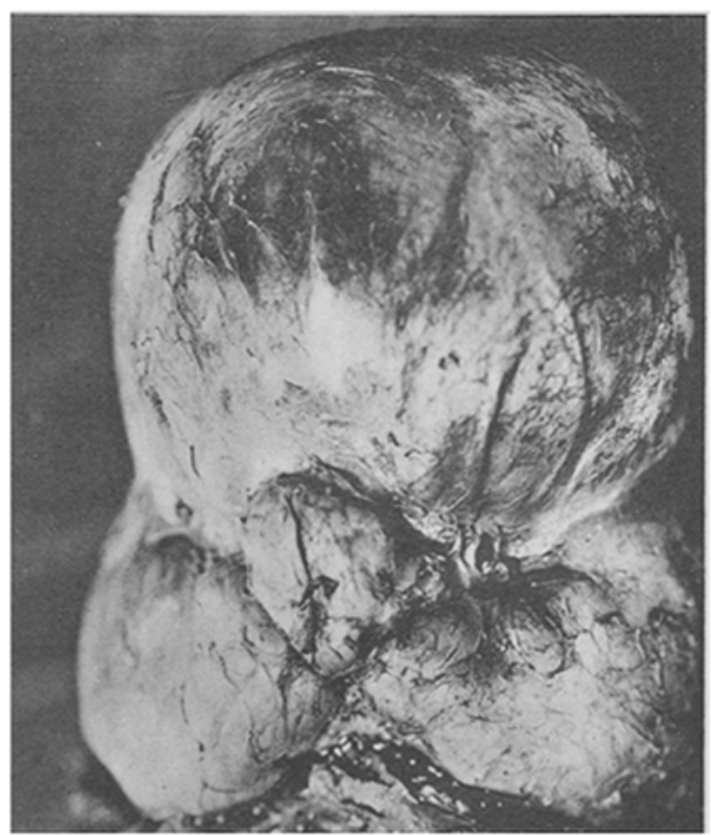

Hydrocephahs internns mit Schnürfurche Occipitalgegend nach Entfernung der Haut und Galea. Kleine Encephalocele occipitalis median. 
Transversalnaht bis zur Promin. occipit. $3 \mathrm{em}$ sagittal und der untere Teil der Schuppe von hier bis zum Foramen occipitale magnum ebenfalls $3 \mathrm{~cm}$ sagittal, also einschliesslich des Spitzenknochens bis zur kleinen Fontanelle $5 \mathrm{~cm}$.

Es bandelt sich hier um einen dreieckigen Schaltknochen, den typischen Repräsentanten des Spitzenknochens.

\section{Viereckiger Schaltknochen der kleinen Fontanelle.}

(Fig. 4, Taf. XVII.)

An dem normal geformten Schädel eines Neugeborenen sehen Sie in der kleinen Fontanelle einen viereckigen rhomboiden Knochen; es fehlt nicht viel zur Form eines Quadrates, doch sind die beiden Seiten, welche den Parietalknochen anliegen, ein wenig länger $(18 \mathrm{~mm})$, als die beiden der Hinterhautschuppe anliegenden Ränder $(15 \mathrm{~mm})$. Der Schaltknochen beeinträchtigt die normale Konfiguration nicht nur des Os occipitale, in welches er mit seiner unteren Spitze vordringt, sondern auch die Form der Seitenwandknochen, da er auch nach oben, scheitelwärts höher hinaufragt, als ein normales os occipitale; man erkennt das daran, wenn man in Verfolgung die Parietooccipitalnähte scheitelwärts verlängert; diese Linien treffen sich, annähernd $1 / 2 \mathrm{~cm}$ unter der kleinen Fontanelle. Der Schaltknochen hat etwa $23 \mathrm{~mm}$ sagittalen Durchmesser und $20 \mathrm{~mm}$ transversalen. Die Ränder des Schaltknochens sind ziemlich glatt, die Nähte sind schmal lamellös.

\section{Zweiteiliger dreieckiger Spitzenkmochen. (Fig. 5, Taf. XVII.)}

Bei einem anderen Neugeborenen mit normal geformtem Schädel. fand ich einen zweiteiligen Spitzenknochen, welcher aus zwei unregelmässigen aber annähernd rechtwinkligen Dreiecken besteht. Die beiden rechten Winkel stossen aneinander. Also der zweiteilige Spitzenknochen bildet als Ganzes betrachtet ein Dreieck, von dessen Spitze eine lotrechte, bereits in Verschmelzung begriffene Naht in der Verlängerung der Sagittalnaht auf seine Basis geht. Die Basis hat $21 / 2 \mathrm{~cm}$ Länge, das Lot etwas mehr als $1 \mathrm{~cm}$, die Seiten des Dreiecks, welche den Parietalnähten anliegen, messen $2 \mathrm{~cm}$. Auch hier ersetzt der Spitzenknochen die fehlende Spitze des Os occipitale, dessen Schuppe von der abnormen Transversalnaht bis zur Protuberantia occipitalis $3 \mathrm{~cm}$ misst, in der Sagittallinie, also einschliesslich des Spitzenknochens bis zur Fontanelle $4 \mathrm{~cm}$.

\section{Zweiteiliger dreieckiger Spitzenknochen der kleinen Fontanelle.}

Der Fetus von 6 Monaten hat eine gut ausgebildete Hinterhauptschuppe, von welcher nur die obere dreieckige Spitze durch eine Transversalnaht abgetrennt und dann noch durch eine Sagittalnaht in zwei annähernd gleiche Dreiecke geteilt ist. An der Basis messen die beiden. Schaltknochen 6 und $8 \mathrm{~mm}$, in der Sagittallinie $5 \mathrm{~mm}$.

\section{o. Spitzenknochen von unregelmässiger Form in der kleinen Fontanelle.}

Bei einem anderen Fetus von 6 Monaten ist ebenfalls ein Spitzenknochen vorhanden, doch ist keine reine Transversalnaht als TrennungsIinie an der Hauptschuppe vorhanden. Yielmehr fehlt an der Dreieck- 
form des Spitzenknochens die rechte Ecke. Durch diesen unregelmässigen Schaltknochen von $8 \mathrm{~mm}$ Breite und $8 \mathrm{~mm}$ Höhe wird mehr das linke Seitenwandbein beeinträchtigt als das rechte,

\section{Kleiner Schaltknochen in der kleinen Fontanelle.}

Bei einem Fetus von 7 Monaten ist ein nur unbedeutender Schaltknochen ron $2 \times 3 \mathrm{~mm}$ Durchmesser gefunden, welcher die übrigens schon sehr weit ausgebildete Hinterhauptschuppe rechts von der Sagittalnaht nur wenig behindert.

Es bandelt sich also in diesen 6 Fällen um Schaltknochen der kleinen Fontanelle, von denen einer beim Fetus ron 7 Monaten nur sehr unbedeutend ist, so dass er vermutlich sehr bald verschwinden würde durch Verschmelzung mit der Hinterhauptschuppe. Einer beim Fetus von 6 Monaten hat nicht die regelmässige Dreieckform und auch hier würde die Transversalnaht vielleicht später verschmolzen sein. In dem Falle 2 ist die Form des Knochens viereckig, seine untere Spitze springt in die Schuppe vor; es ist das jene schon von Virchow erwähnte Form, welche er nicht dem Spitzenknochen zurechnen wollte. Zwei Feten, ein 7 monatlicher und ein ausgetragenes Kind haben dreieckige, aber durch eine Sagittalnaht geteilte Schaltknochen, was ebensowenig wie die viereckige Form gegen die Zugehörigkeit zu den Spitzenknochen spricht. Im Gegenteil erscheint bei diesen die Sagittalnaht als das ursprünglichere Zeichen der Entstehung aus dem atypischen Knochenpaare 4 in dem Entwicklungsschema Rankes. Diese Sagittalnaht verwächst scheinbar am schnellsten in unseren Fällen.

Aber auch die übrigen Nähte würden wohl nicht lange der Vereinigung widerstanden haben. Anders der an erster Stelle genannte Schaltknochen beim Neugeborenen; dieser aussergewöhnlich grosse Spitzenknochen ist durch eine breite Naht von der übrigen Hinterhauptschuppe getrennt, die Naht wird durch 1-2 mm breite, durchsichtig dünne Membranen dargestellt, welche nur von einzelnen feinen Ausläufern rom zackigen Rande des Spitzenknochens her durchzogen wird. Dieser grosse Sehaltknochen würde vermutlich der Vereinigung widerstanden haben und eine richtige zackige Knochennaht hinterlassen haben. Bei Fall 2 lässt sich kein Urteil fällen über das vermutliche weitere Schicksal; hier fiel besonders auf, dass der Schaltknochen eine dunklere Färbung hat, was von seiner porösen zarten Natur herrührt. Auch die übrigen Schaltknochen haben ausser Fall 1 ein poröses, 
weniger konsolidiertes Aussehen. Im Fall 1 erscheint der Schaltknochen nicht weniger solide, als die anderen Schädelknochen. Bei jüngeren Feten habe ich ebensowenig Schaltknochen der Fontanellen gesehen, wie Ranke.

Ich lege Thnen schliesslich noch mit freundlicher Erlaubnis des Herrn Carl Ruge 3 Fälle von Spitzenknochen vor, welche ich in der Schädelsammlung der Universitäts-Frauenklinik vorfand, und von denen einer an Grösse und an Breite der Nähte meinem Fall 1 gleicht, mit dem Unterschiede, dass er sich in unregelmässiger Weise mehr der viereckigen Form nähert. Sie ersehen, dass die verschiedenen Formen der Spitzenknochen bedingt sind durch die verschiedene Art der Transversalnaht; ist diese annähernd gradlinig, so ist die Form dreieckig; von der gradlinigen bis zur winkligen Transversalnaht beim ausgesprochenen viereckigen Spitzenknochen gibt es alle Uebergänge, so dass hier kein prinzipieller Unterschied aufgestellt werden kann, wie Virehow meinte. Man darf annehmen, dass die Form des Spitzenknochens lediglich davon abhängt, nach welcher Seite hin die normalen Knochenkerne ihm Platz lassen; ich meine hiermit, dass die Form des Spitzenknochens nicht etwa von vornherein in seiner Anlage bedingt jst, sondern dass er nur dort sich ausbreiten kann, wo die Parietalbeine und die typischen Kerne des Occipitalbeins im Wachstum zurückbleiben. In der vorderen Fontanelle habe ich nur einige Male Schaltknochen gesehen, von denen wir noch sprechen werden, aber diese unterscheiden sich wesentlich von denen der hinteren Fontanelle durch ihre diffuse Verknöcherung der Fontanelle, so dass man nicht den Eindruck eines selbständigen Knochenkernes hat, wie bei den hinteren Fontanellknochen; und gerade dieses umschriebene Auftreten an einer bestimmten Stelle der hinteren Fontanelle deutet auf eine Bildung, welche in der Entwicklung zwar nicht typisch, aber doch auch nicht pathologisch, sondern höchstens mit Ranke haibpathologisch bezeichnet werdon darf. Jedenfalls liegt hier eine phylogenetische Erscheinung vor, sei cs eine atapistische oder eine Neuerscheinung, welche der Zukunftsnorm angehört.

Veine Herren, ich habe Ibnen diese Anomalien der hinteren Fontanelle, welche anatomisch so ausserordentlich oft behandelt wurden, nur aus dem Grunde vorgelegt, weil es mich erstaunt, dass ein solch auffallender Befund nicht bereits geburtshülflich erkannt wurde. Meiner Meinung nach müssto man fast ebensogut 
wie die normale Fontanelle auch eine solche mit grösseren Schaltknochen palpatorisch erkennen können. Es scheint mir deshalb die Frage berechtigt, ob in einem Falle wie dem vorliegenden (Fall 1) nieht Fehldiagnosen der Kindslage dadurch entstehen können, dass man eine der beiden basalen Ecken des Schaltknochens für die Fontanelle anșpricht.

Ich glaube nicht, dass man diese Frage sogleich beantworten kann, und möchte deshalb bitten, wern aus der Palpation entstandene diagnostische Irrtümer nachher erkannt werden, an die Schaltknochen zu denken, welche bei Niengeborenen in etwa 6 pCt. vorkommen.

Schliesslich zeige ich Ihnen noch einen Fall mit multiplen, ungewöhnlich starken Nahtzwickelknochen der Lambdanaht und einen Fall ron Inkabein, der allerdings keinen ganz normalen Kopf betrifft; es handelt sich um cinen, wie Sie sehen, sonst vorzüglich ausgebildeten Schädel eines Neugeborenen mit mässigem Grade von Hydrocephalus, mit einer Transversalnaht, welche den grösseren 'Teil der Oberschuppe rom übrigen Schuppenteil breit trennt, so dass hier ein richtiger Intraparietalknochen solbständig auftritt (s. Fjg. 6, Taf. XVIII).

\section{Anomalien der vorderen Fontanelle. Anomalien der Grösse und Verknöcherung.}

Der zweite Teil meiner Demonstration soll ganz kurz die individuell verschiedene Form und Grösse der Fontanelle vor Lugen führen, welche wohl ebenfalls einige Beachtung seitens der Geburtshelfer verdient, sowie die Terknöcherung der Vorderfontanelle. Die hintere Fontanelle wechselt sehr viel weniger an Form und Grösse als dic vordere; an der hinteren Fontanelle ist nur die Winkelbildung variabel; manchmal steigen die Ränder der Hinterhauptschuppe steil an zu einem rechten Winkel, manchmal bilden sie mehr einen Bogen, welcher sich der Kreisform nühert. Die Hinterhauptschoppe fült zuweilen schon mit 6 Monaten den ganzen intraparietalen Raum aus, seltener scheint eine zu srosse Hinterhauptsfontanelle beim Neugeborenen vorzukommen.

Viel bedeutender sind die Schwankungen in der Grösse und damit zugleich in der Form an der vorderen Fontanelle; Sie seben hier ziemlich extreme Fälle, einerseits sehr kleine Fontanellen am Vorderhaupt bei Feten von 7 und 6 und sogar schon 
von $51 / 2$. Monaten und andererseits riesige Fontanellen beim ausgetragenen Kind und beim Fetus von 5 Monaten.

Bei einem Fetus ron $5^{1 / 2}$ Monaten, dessen Schädel an den Stimbeinetr einen Querdurchmesser von $48 \mathrm{~mm}$ und an den Seitenwandbeinen ron $56 \mathrm{~mm}$, und einen grössten Längsdurchmesser von $65 \mathrm{~mm}$ hat, sind die Seitenwandbeine schon an den vorderen medianen Ecken fast rechtwinklig auscebildet; die vordere Fontanelle liegt daher fast nur zwischen den Stirnbeinen und da auch diese schon nahe gegen einander getreten sind, so bleibt nur eine Fontanelle von geringer Grösse übrig, etwa $7 \times 7 \mathrm{~mm}$ grösster Durchmesser.

Bei einem Fetus von 6 Monaten sind es die Stirnbeine, welche auffallend weit gediehen sind, aber auch die Seitenwandbeine sind gut ausgebildet, so dass nur eine Bautengrube von $6 \mathrm{~mm}$ grösster Breite und $9 \mathrm{~mm}$ grösster Länge besteht.

Bei einem Fetus von 7 Monaten liegen die Verhältnisse ähnlich wie bei dem von $5 \frac{1}{2}$ Monaten. Die Fontanelle liegt hier fast nur zwischen den Stimbeinen als länglicher, schmaler Spalt.

Ebenso ist hier bei einem Neugeborenen die vordere Fontanelle nur minimal zu nennen (Fig. 7, Taf. XVIII). Die geringe Grösse der Vorderhauptfontanelle beruht also, wie sie sehen, auf einem frühzeitigen Auswachsen der Stimbeine und Seitenwandbeine, welche nicht wie gewöhnlich mit weit geschweiften Rändern zusammen eine grosse rautenförmige Lücke hinterlassen; vielmehr haben die Knochen schon rechtwinklige und spitzwinklige Ecken, welche nahe an einander zu liegen kommen, so dass die Fontanelle erheblich reduziert und in Form einer unregelmässigen Lücke erscheint. Für diese frühreife Knochenentwicklung weiss ich keine Ursache anzugeben; nicht einmal die mechanische Ursache eines geringen Schädelinhalts war nachweisbar. - Eine grosse geburtshilfliche Bcdentung wird dieser Anomalie kaum zukommen. Ihr gegenüber steht die übermässig grosse Fontanelle, welche uns zu den Defektbildungen der Schädeldeckknochen überleitet. Ich stelle also hier die übergrosse Fontanelle vor, bei welcher keine der sonst bekannten Lrsachen für Defektbildung nachweisbar ist; es handelt sich vielmehr um eine geringe Ausbildung der die vordere Fontanelle umgebenden Deckknochen, ohne dass eine lokale oder allgemeine Ursache der Störung erkennbar wäre.

Ein auffälliger Befund derart ist mir bisher nur einige Male passiert; der normal geformte Schädel dieses 8 Monate alten Fetus hat grössten Breitendurchmesser an der Stirn von fast $60 \mathrm{~mm}$, an den Seitenwandbeinen von etwa $80 \mathrm{~mm}$ und etwa $90 \mathrm{~mm}$ Längsdurchmesser. Die Hinterhauptfontanelle ist noch nicht ganz mit Knochen erfüllt; immerhin ist sie nicht anffällig gross, während die grosse Fontanelle sich durch eine besondere Grösse fast $45 \times 45 \mathrm{~mm}$ auszeichnet. Der Knochen- 
defekt kommt noch mehr auf die Stimbeine, als auf die der Seitenwandbeine, doch sind auch diese reichlich beteiligt. Die Schädelknochen weichen im übrigen nicht von der Norm ab. Die grosse Fontanelle besteht aus einer teilweise sehr dünnen Membran, welche jedoch ganz unregelmässig in sehr dünner Lage in grosser Ausdehnung zu verknöchem beginnt. Bei einem Fetus von 6 Monaten sind die Deckknochen des Schädeldaches sehr dünn. besonders die Stirnschuppen, die an vielen Stellen durchsichtio sind; letztere sind sehr zurückgeblieben, aber auch die Seitenwandbeine, so dass die vordere Fontanelle $5 \times 5 \frac{1}{2} \mathrm{~cm}$ Durchmesser hat bei einem grossen Querdurchmesser des $6^{1 / 2} \mathrm{~cm}$. Die Sagittalnaht klafft zwischen den Seitenwandbeinen vorne 8 , hinten 10-19 $\mathrm{mm}$ breit. Im übrigen ist der Schädel normal, und es fanden sich auch sonst keine Anomalien. Diese zarten Knochenmassen liegen zum Teil frei in der Membran, zum Teil grenzen sie an die Ränder der normalen Schläfenknochen.

Es ist also hier bemerkenswert einerseits die zurückgebliebene Ausbildung der regulären Knochen, andererseits der ausgedehnte Ersatz durch diffuse fontikuläre Verknöcherung. Es liegt also keine allgemeine Störung chemischer Art, keine qualitative Anomalie vor, welche die Knochenbildung bintanhielte, ebensowenig sind lokale mechanische Ursachen, wie Hydrocephalus oder ein Tumor nachweisbar, welche durch Dehnung die Knochenentwicklung quantitativ hemmen. Der Schädel ist sonst auch normal ausgebildet, so dass. man nur eine lokale Schwäche der betr. Knochenkerne besonders. der Stirnbeinschuppen annehmen kann; also eine quantitative Defektbildung (Schwalbe) ohne nachweisbare Ursache an den Deckknochen.

In einem auffälligen Gegensatze dazu steht die Verknöcherung: der Fontanelle in diesem Falle; sie ist bemerkenswerter Weise ganz unregelmässig und diffus (Fig. 8, Taf. XIX).

Auch bei nicht so besonders grossen Vorderhauptfontanellen kommt dieser Gegensatz vor, wie ich ihnen ebenfalls beim letus von 8 Monaten zeigen kann, bei welchem die Fontanelle etwa $25 \times 25 \mathrm{~mm}$ grössten Durchmesser hat und in ganzer Ausdehnung vor zarter Knochenmasse diffus erfïllt ist.

Bei einem Fetus von 7 Monaten ist die Fontanelle am Vorderhaupt noch kleiner; $21 \mathrm{~mm}$ lang, $17 \mathrm{~mm}$ breit. Sie ist normal geformt und nur der: hintere Teil zwisehen den Seitenwandbeinen ist durch eine ganz unregelmässig geformte diffuse Knochenbildung in diinner Lage fast ausgefuillt; die Knochenlamelle geht in die Seitenwandbeine soeben über; besonders in das linke und erreicht mit einigen Fasern auch das linke Stirnbein. Nach vorne zu setzt sich der verknöcherte Teil der Fontanelle mit unregelmässigen Ausstrahlungeu ganz unscharf gegen die häutige Lamelle ab.

Die Verknöcherung der vorderen Fontanelle fand jch immer: diffus und ganz unregelmässig, also keineswegs an besonders lokalisierte Knochenkerne gebunden, wie die schärfer umschriebenen 
Schaltknochen der hinteren Fontanelle; diese sind wie gesagt analog den Spitzenknochen einiger Tiere, während in der vorderen Fontanellgegend weder typische noch atypische Knochenkerne bekannt sind.

\section{Hypoplasie, Aplasie und Defekte der Schädel= deckknochen.}

Weine Herren! Mir sind drei ursächlich verschiedene Arten von Defekiten der Schädelknochen vorgekommen; nämlich Defekte bei Osteogenesis imperfecta, zweitens infolge äusserer Schädigung, und drittens durch Hydrocephalus. Die zweite und dritte Art kann man als mechanische Schädigung zusammenfassen gegenüber der ersten Art von chemischer Störung. Ich spreche hier, um das ausdrücklich za betonen, nicht von Defekten der ganzen Schädeldecken, wie wir sie bei Hemicephali und partiellen, wie wir sie bei Kephalocelen kennen.

\section{Völlige Aplasie der Schädeldeckknochen bei Osteogenesis imperfecta.}

Ich zeige Ihnen einen Fetus im 8. Monat, bei welchem ausser etwas Lrummen Extremitäten mit kurzen Vorderarmen und Unterschenkeln nur ein etwas vergrösserter Kopf auffällt. Bei normaler Gesichtsbildung erscheint der Schädelteil des Kopfes reichlich gross; $30 \mathrm{~cm}$ Limfang. Der Kopf ist auffallend rundlich und so weich, dass man im Liegen nicht die Durchmesser richtig messen kann, weil er sich dann zusammendrängt. Der grösste Querdurchmesser beträgt etwa $10 \mathrm{~cm}$ und ebenso der Längsdurchmesser. Alle Knochen fühlen sich weich an, sowohl an den Gliedern, wie auch am Rumpf und im Gesicht. Am Schädeldach fühlt man jedoch keinerlei Knochen, auch im Röntgenbilde erscheint keine Spur und auch nach Abpräparieren der Kopfhaut ist keine Spur von Deckknochen nachweisbar. Man fühlt zwar die Lnterschuppe des Hinterhauptbeins durch und das Os petrosum, aber die Schuppen des Felsenbeins und des Stirnbeins, die Seitenwandbeine und die Oberschuppe des Hinterhauptbeins fehlen vollständig.

An den Knochen der Extremitäten ist die mangelhafte Knochenbildung viel erheblicher, als man nach der äusseren Konfiguration erwarten sollte. Die Epiphysenknorpel sind gut ausgebildet und grenzen sich gegen die Diaphysen scharf ab. Es fehlt völlig die periostale Knochenbildung. Die Diaphysen bestehen aus einer bräunlich roten, wenig harten bröckligen Spongiosa. 
Im übrigen sind weder äusserlich noch innerlich Missbildungen zu entdecken.

Es handelt sich also hier um einen Fall von Osteogenesis imperfecta; unter diesem Namen versteht man einen Teil derfrüher als fetale Rachitis bezeichneten Knochenerkrankungen im Gegensatz zur Chondrodystrophia fetalis (Kaufmann). Der Unterschied zwiscben beiden Erkrankungen ist bedeutend, indem bei Chondrodystrophie die enchondrale Ossifikation leidet, während die endostale und perichondrale Ossifikation normal sein, letztere sogar gesteigert sein kann. Umgekehrt ist bei der Osteogenesis imperfecta die enchondrale Knochenbildung normal, dagegen die endostale und perichondrale Knochenbildung gestört, und da die Schädeldeckknochen nicht knorpelig vorgebildet sind, so leiden diese am auffälligsten bezw. fehlen wie in unserem Falle gänzlich.

Ich werde Ihnen kurz die normale Knochenentwicklung und die gestörte bei Ostcogenesis imperfecta und Chondrodystrophie in Lumière-Mikrophotogrammen zum Vergleiche vorführen. Von der letzteren Erkrankung haben wir mehrere Fälle in der Charité gesehen. Dic letzten Beiträge zu der Lehre von den fetalen Knochenerkrankungen mit besonderer Berücksichtjgung der anatomischen und klinischen Differentialdiagnose und Uebersicht über die Literatur finden Sie bei Sumita, einem Sehüler K Kaufmanns (Deutsche Zeitschr. f. Chir. 1910. Bd. 104). Die auffallendsten äusseren Unterschiede zwischen beiden Erkrankungen, welche Kaufmann und Sumita angegeben haben, finden Sie auch an den vorliegenden Exemplaren. Diese sind nach Sumita:

Bei Chondrodystrophie: Aufiallende Mikromelie. Gut verknöcherter, grosser Kopf.

Häufige prämature Synostosen. Häufige Nasenwurzeleinziehung. Viereckige und dreizackige Hände. Feste massive Diaphysen.

Zackige Ossifikationslinie.

Vorhandensein des Perioststreifens.

Gestörte enchondrale Ossifikation. Ungestörte periostale und endostale Ossifikation.

Fast immer normale Clavicula. Häufige Kombination mit Missbildungen.
Bei Osteogenesis imperfecta:

Weniger auffallende Mikromelie.

Weniger umfangreicher, weicher Kopf.

Fehlen der Synostosen.

Fehlen der Nasenwurzeleinziehung.

Feine zarte Hände.

Schwache, brüchige Diaphysen.

Gerade regelmässige Ossifikationslinie.

Fehlen des Perioststreifens.

Normale encondrale Ossifikation.

Gestörte periostale und endostale Ossifikation.

Nicht geschonte Clavicula.

Seltene Kombination mit anderen Missbildungen. 
Die genannten Erkrankungen haben auch praktisches Interesse, da Kinder mit leichteren Graden lebensfähig sind. Zwar sind bisher Aetiologie und Therapie unbekannt, dennoch muss natürlich auch der Praktiker die Krankheitsbilder kennen. Besonders wichtig würde es wohl sein, auch die leichtesten Grade vielleicht an der mangelhaften Verknöcherung der Schädeldecke, an einer zu grossen vorderen Fontanelle zu erkennen. In dieser Richtung liegt noch ein Arbeitsfeld frei.

Hier liegt der Schädel eines Neugeborenen vor, bei welchem die Knochen eine normale Flächenausdehnung haben, aber eine geringe oder eine schwache Vorkalkung, denn sie waren besonders in der Näbe der Pfeilnaht so biegsam, dass sie beim Tasten nachgaben. Herr Kollege Zinsser hat ebenfalls einen so weichen Kopf vor liurzem beobachtet, welcher unter der Geburt lagediagnostische Schwierigkeiten machte; auch nach der Geburt war der Schädel weich, wurde aber nach wenigen Tagen, wie ich mich überzengen konnte, von normaler Härte. Ob solche Fälle hierher gehören, kann ich nicht sagen.

\section{Schädelknochendefekte durch äussere Trsachen; Schädignug des Kopfes durch die Eihäute, die Placenta oder die Iteruswand.}

Die zweite Art von Defekten der Schädeldeckknochen ist jedenfalls selten; sie kommt durch äussere Läsionen zustande; solche Läsionen werden beobachtet bei Verwachsung der Eihäute oder der Placenta mit dem Kopfe, wie in dem hier vorliegenden Falle, seltener bei Graviditas extrachorialis. Auch einen solchen Fall lege ich Ihnen vor, welchen Ihnen Herr Haendly schon einmal demonstrierte. Freilich kommt es in diesen Fällen nicht nur zur Defekibildung an den Schädeldeckknochen, sondern auch zu. Wachstumsstörungen in den übrigen Schädeldecken, namentlich der Haut. Bei unserem Falle ron extrachorialer Gravidität sind die Schädeldecken auf dem Scheitel bis auf eine ganz dünne Membran reduziert, welche aussen ein sehr reduziertes Pflasterepithel und innen eine einschichtige flache endothelartige Bekleidung trägt; dazwischen ist nur fibrilläres Bindegewebe enthalten mit Blutgefässen; sonst keine Spur der normalen Gewebe.

Wie weit etwa Schädeldefekte bei Anencephalie und Kephalocelen zu den traumatisehen gehören, möge hier unerörtert blciben.

Die traumatischen Schädelknochendefekte aus äusseren $\mathrm{Cr}_{\mathrm{r}}$ sachen haben wohl am wenigsten Interesse. 


\section{Schädelknochendefekte bei Hydrocephalns. Eine besondere Art von Hydrocephalus durch Lmschnïrung des Schädels.}

Bei fast jedem Hydrocephalus findet man grössere oder kleinere Lücken zwischen den Schädelknochen. Ich kann Ihnen sogar den Schädel eines 5 Monate alten Fetus mit mässigem Hydrocephalus zeigen, bei dem der Scheitel fast nur fonticulär erscheint, und die Ränder der Stirnbeine stehen bis zur Nasenwurzel und ebenso die Ränder der Seitenwandbeine bis zur hinteren Fontanelle breit auseinander. Der rautenförmige Defekt misst an der breitesten Stelle über $40 \mathrm{~mm}$ und in der Sagittallinie fast $50 \mathrm{~mm}$, während der ganze Schädel nur $47 \mathrm{~mm}$ breit und $60 \mathrm{~mm}$ lang ist. Zarte Knochenbälkchen durchziehen in geringer Menge einzelne Stellen der Fontanelle, besonders im vorderen Teile.

In diesem Falle kann man wohl von einem wirklichen Defekt sprechen, da die Deckknochen auch zur Bedeckung eines normalen Schädels nicht ausreichen würden, und man muss annehmen, dass der Schädelbinnendruck die Knochenbildung beeinträchtigt. Es liegt aber in solchen Fällen nicht immer ein wirklicher Defekt vor, vielmehr ist die Flächenausdehnung der Knochen oft recht erheblich, ohne jedoch den abnormen Ansprüchen gerecht werden zu können, und auch in den fonticulären Liicken treten oft zahlreiche Verknöcherungsherde auf, wie in dem vorliegenden Falle bei einem Neugeborenen mit erheblichem Grade von Hydrocephalus. Nur in einem Falle habe ich auch wirkliche Knochendefekte am Schädel gefunden und dieser Fall ist auch sonst so eigentümlich, dass es wohl lohnt, ihn genauer zu betrachten. - Wie Sie beim ersten Anblick sehen, handelt es sich um eine ganz ungewöhnliche Form von Hydrocephalus, denn während sonst, wie ein Vergleichsfall zeigt, das kleine Gesicht gegen den grossen Schädel absticht, ist zwar hier der Schädel anch vergrössert, aber das Gesicht erscheint noch mebr vergrössert. (Fig. 9 u. 10 auf Taf. XIX-XX.)

Die Mutter, 24 Jahre, primigravida, normal gebaut, gibt als Termin der Empfängnis den 2. September 1910 an. Sie fühlte Ende Februar 1911 die ersten Kindsbewegungen. Sie merkte keine Senknng der Gebärmutter. Am 13. März wird sie mit Wehen in die Frauenklinik der Charité gebracht. Die Wehen begannen $10 \mathrm{Uhr}$ vormittags, bei der ersten Untersuchung ist der Kopf schon sichtbar, der Blasensprung erfolgte 7 Uhr nachmittags, die Geburt 7 Uhr 20 Min. Die Geburt erfolgt spontan. Das Kind lebend, stirbt nach einigen Minuten. Frucht- 
wasser ohne Besonderheit. Nabelschnur normal, inseriert an der normalen Placenta.

Der männliche Fetus hat $40 \mathrm{~cm}$ Länge, davon beträgt die Kopfhöhe allein $17 \mathrm{~cm}$; der Kopf hat einen geraden Durchmesser von 12, einen schrägen von $12^{1 / 2} \mathrm{~cm}$ und $10 \mathrm{~cm}$ Querdurchmesser sowohl parietal als frontal. Der Kopf von vorn betrachtet, hat Kürbisform, unten breiter als oben; der untere Gesichtsteil bis über die Augen ist nämlich umfangreicher und vor allem breiter $(12 \mathrm{~cm})$ als die Stirn und Schädeldachpartie $(10 \mathrm{~cm})$, von welcher sie sich durch eine breite Furche absetzt. Die Furche geht zirkulär um den Schädel, ist am deutlichsten an den Schläfen- und Parietalbeinen, weniger dentlich über den Augen, in der Gegend der Supraorbitalränder, und ebenfalls undeutlicher etwa in der Gegend der kleinen Fontanelle. Der Kupi soheidet sich auf den ersten Blick in zwei Partien (Fig. 9 u. 10), die untere Gesichtspartie bis über die Augen, die Schläfen- und Hinterhauptsgegend bilden zusammen den breiteren umfangreicheren Teil ( $33 \mathrm{~cm}$ Umfang), die Stirnund Seitenwandbeine den oberen. Teil mit $31 \mathrm{~cm}$ Umfang. Im Gesicht fällt neben der abnormen Breite auf, dass die Augen vorstehen, nicht etwa nur, weil die Orbitalränder zurückgedrängt erscheinen in der Furche, sie quellen über die Gesichtsoberfiäche hervor, die Lidspalten sind schlitzförmig geöffnet, die Schlitze lateral tiefer konvergieren gegeneinander nach oben zur Nasenwurzel hin, so dass ausser dem Exophthalmus noch ein besonderer clownhafter Ausdruck entsteht. Die Breite des Gesichts wird dadurch erzielt, dass die Schläfenpartie heruntergedrängt ist, so dass die Backen durch die Schläfen verbreitert werden und die fast horizontal gestellten Ohrmuscheln (Fig. 10) mit dem unteren Kinnrande eine Linie bilden; dementsprechend bildet auch der Unterkiefer einen langen horizontalen Ast, keinen normal aufsteigenden Ast; deshalb sind auch die Augen schräg gestellt, aussen tiefer als medial. Nase und Mund sowie alle Organe erscheinen normal bis auf eine Gaumenspalte, von welcher der weiche und der hintere Teil des harten Gaumens betroffen wird. Aber die zu tief liegende Schläfenpartie, welche auch sonst bei Hydrocephalen vorkommt, wenn auch nicht so. extrem, würde allein nicht genügen, das Gesicht so verbreitert erscheinen zu lassen; es kommt hinzu, dass die Schlälengegend aufgebauscht ist. Von den $17 \mathrm{~cm}$ Kopfhöhe kommen nur $5^{1 / 2} \mathrm{~cm}$ auf die Höhe von der Kinnspitze bis zur Nasenwurzel, dagegen liegt seitlich über den Schläfenbeinen die Furche etwas höher, so dass im Profil die obere Kopfpartie nicht so hoch erseheint. Am Hinterhaupte fällt eine merkwiïrdige Buckelung auf; zwei seitliche Buckel, und darüber ein mittlerer Buckel bilden scheinbar die Hinterhauptschuppe.

Bei Abtastung des Schädels ergibt sich eine höchst ungleichmässige Verteilung der Schädelknochen und ein starkes Ballottement, welches am merkw ürdigsten, weil am ungewöhnlichsten sich ausnimmt in den abnorm tie? gelegenen Schläfen; man löst hier dem Anscheine nach in der Gesichtsgegend, in Wirklichkeit freilich von den aufgebauschten Schläfen her das Ballottement am Scheitel aus. Die Gesichtsknochen sind von normaler Härte, die Stimbeine sind nur in der Supraorbitalgegend konsistent, darüber sind die Schuppen als sehr dünne biegsame Lamellen zu füllen, zwischen denen eine grosse breite Lücke vöIlig knochenfrei bleibt. Da auch die Seitenwandbeine nicht viel besser ausgebildet sind, so bleibt fast der ganze Scheitel ohne knöcherne Decke, es besteht sozusagen eine riesige Fontanelle, welche bis in die Nähe 
des Hinterhauptknochens reicht; hier hinten sind die Parietalbeine besser zu fühlen. Die 3 Buckel der Hinterhauptsschuppe fühlen sich am derbsten an. Auch die Schläfenbeinsehuppen knittern bei der Betastung. und sind besonders dünn. Die Röntgenaufnahmen ergeben keine so guten Resultate wie die Betastung des Kopfes.

Auf dem Röntgenbilde im Profil ist die Projektion der Schläfenknochen auf das Gesichtsskelett so verwirrend, dass man ohne vorherige. Kenntnis der Ursache kaum auf eine richtige Deutung kommen kann. Nach Lospräparieren der Kophant bestätigt sich das itber die Schädelknochen Gesagte; die in Formalin gallerartig gewordene Kopfgeschwulst wird entfernt und nun zeigt sich an der kleinen Fontanelle eine Lücke. in welcher sich eine kleine Encephalocele befindet. Die Hinterhauptschuppe besteht aus höchst unregelmässigen Buckeln, von denen die drei grössten bereits bei der Betastung auffielen (Fig. 11, Taf. XX).

Nach Eröffnung des Schädels quillt die Gehirnmasse vor, welche in den Ventrikeln eine Flüssigkeitsmenge enthält, nicht mehr als bei einem mässigen Grade von Hydrocephalus. Das Schädelinnere entspricht der äusseren Form, insofern die Basis stark deformiert ist; namentlich auffällig ist innen die unregelmässige Ausbeulung des Hinterhauptbeines und mit kurzen Worten die ausserordentliche Vertiefung der mittleren Schädelbasispartie (Fossa crania media) gegenüber der vorderen; insbesondere die Verdrängung der Schläfenbeine in basaler Richtung. An Stelle der mittleren Schädelhöhle ist eine enorme Ausbuchtung mit Deformation der sie bildenden Knochen vorhanden, welche zum geringeren Teile das Keilbein betrifft. Die hinteren Ränder der kleinen Keilbeinflügel springen äusserst scharfkantig und flach nach hinten vor und unter ihnen buchtet sich das Keilbein sehr tief nach vorne unter Verdrängung der grossen Keilbeinflügel. Da nun das Schläfenbein seinerseits enorm nach unten verdrängt ist, so ist eine enorme Knochenlücke vorhanden zwischen Schläfenbein einerseits und Keilbein und Oberkiefer andererseits. Die Schläfenbeinschuppe ist aber, abgesehen von der relativen Unfähigkeit, die abnorm tiefe mittlere Schädelgrube zu decken, auch absolut zu klein geraten und zu dünn. Dadurch wird es also möglich, zu Seiten des Kinns und der Wangen, wie oben gesagt, Ballottement im Gehirn zu erregen, die mittlere Sehädelgrube ist basal nur mit stark gedehnten und verdünten Weichteilen versehen und ragt so aussergewöhnlich tief, dass sie das Gesicht in der Kinngegend verbreitert.

Das Wesentliche, was diesen Fall von Hydrocephalus von der gewöhnlichen Form unterscheidet, ist äusserlich betrachtet, die Zweiteilung des Kopfes in eine obere und untere durch eine Furche getrennte Partie und vor allem die starke Vergrösserung des Gesichts, welche bedingt wird durch Versetzung der Schläfenpartien mit den Ohren nach unten an die Seite der Wangen und des Kinns unter Auftreibung der Schläfenschuppengegend zu Seiten der Wangen. - Wie schon gesagt, kommt bei stärkeren Graden der Hydrocephalie auch der Druek auf die Schädelbasis aussen zur Geltung durch Tiefertreten der Schläfengegend und der Ohren und auch am macerierten Schädel beim Hydrocephalus sieht man das Trommel- 
fell mehr basal gestellt, aber durchaus nicht immer, wie der Vergleich mit viel stärkeren Graden von Hydrocephalus lehrt, und nicht in so extremer Weise wie hicr. Exophthalmus und Gamenspalte sind auch beim gewöhnlichen Hydrocephalus stärkeren Grades bekannte Erscheinungen. Wenn wir die Lrsache dieser besonderen Art von Missbildung suchen, so ist wohl kaum darar zu zweifeln, dass der Hydrocephalus als soleher, d. h. die Wasseransammlung allein nicht die Schuld an der abnormen Form des Kopfes sein kann, vielmehr kann man ohne weiteres behaupten, dass eine mechanische Ursache Schuld tragen muss an der Zweiteilung des Kopfes. Die zirkuläre Furche um den Kopf, welche den oberen kleineren Toil des Hydrocephalus trennt gegen den Gesichtsteil, ist gar nicht möglich anders aufzufassen, da die Furche keinerlei Phasen der normalen Entwicklung entspricht. Sämtliche Erscheinungen lassen sich leicht erklären durch eine Jmschnürung des oberen Kopfteiles, welcher infolgedessen nicht wie sonst bei der Wasseransammlung in den Ventrikeln nach allen Seiten an Ausdehnung gewinnen konnte. Die Expansion wendete sich daher mehr dem unteren Teile des Schädels zu und verursachte die ungewöhnliche Gesichtsform.

Alles, was unter der Schnürfurche des Schädels liegt, Hinterhauptschuppe und mittilerer Teil der Schädelbasis hat unter dem Drucke stärkere Dilatation und Deformation erlitten, als der nach unserer Annahme allseitig umschnürt gewesene obere Kopfteil.

Jetzt erhebt sich die Frage, wie eine dauernde Unsschnürung und Beengung des oberen Koptteils zu Wege kommen kann. Da es sich um eine Sohädellage bei der Geburt handelte, so ist anzunehmen, dass der Schädel im unteren Teile der Gebärmutterhöhle fest umschnürt längere Zeit gelegen haben muss. Wie lange die Umschnürung angedanert hat, lässt sich kaum genauer bestimmen, doch darf man den Termin recht früh annehmen, weil vor allem die Gaumenspalte aber auch die Deformation der Schädelhöhle und dic Knochendefektbildung in späteren Stadien nicht gut entstehen kann. Wir müssen also annehmen, dass die Wasseransammlung im Gehirne im zweiten Monate schon eine bedeutende Ausdehnung des Kopfes verursacht hat und dass irgend eine Besonderheit seiner Umgebung also entweder der Fihäute oder der Uteruswand den oberen Kopfteil umschnürt hielten.

Eine circumscripte Einengung durch das Amnion kann ich mir kaum vorstellen, dazu ist das Amnion auch nicht fest genug; 
ebenso glaube ich nicht, dass ohne besondere Verwachsung mit Eihäuten die Sachlage erklärlich wird und das würde man sehen müssen. Zu erörtern wäre kaum eine Umschnürung des Kopfes durch die Nabelschnur, denn es handelt sich nicht um eine Sehnürfurche, auf deren beiden Seiten der Kopf einen stärkeren Umfang hat, als in der Furche selbst. Vielmehr ist das ganze Oberhaupt von der Schnürfurche ab dünner als der Gesichtsteil und das ist nur zu erklären durch eine Umschnürung des ganzen Oberhauptes bis zur Schnürfurche.

Man kann sich nun verschiedene Vorstellungen machen, wie eine solche Einengung des Kopfes zustande kommt; der obere Kopfteil könnte in einem Divertikel der Uteruswand gelegen und hierdurch einen Wachstumswiderstand erfahren haben. Diese Annahme ist an und für sich nicht bedenklich, da ich ein analoges Beispiel anführen kann. Herr Olshausen hat in dieser Gesellschaft (24. Juni 1902) einen exstirpierten Uterus vorgestellt mit einem grossen Divertikel an der Hinterwand unten, welches bei der Untersuchung vor der Operation für ein Myom gehalten wurde. Das abgestorbene Kind lag in Steisslage im Uterus und ein durch Umschnürung abgegrenzter Teil des Steisses füllte das Divertikel genau aus. Herr Olshausen stellte es als eine Sakralgeschwulst vor; da mir aber die merkwürdige Anpassung der Geschwulst in das Divertikel für einen ursächlichen Zusammenhang beider unbedingt zu sprechen schien und ich mir nicht vorstellen konnte, dass ein Kindsteil ein Wanddivertikel bildet, noch weniger, dass ein Divertikel eine Geschwulst am Kinde hervorruft, so untersuchte ich nachträglich mit gütiger Erlaubnis des Hern O. Ruge das Präparat. Ich konnte feststellen, dass es sich nicht um eine Gesehwulst handelte, sondern um einen abgeschnürten Teil des Kindsteisses, durch welchen ein Teil des stark dilatierten Rectum verlief. Jetzt war die Sachlage verständlich geworden: der Steiss war in jenes präformierte Divertikel geraten und wurde beim weiteren Wachstum des Kindes teilweise im Divertikel retiniert und eingeengt bzw. teilweise abgeschnürt.

Ich kann in diesem Falle nur ein Beispiel für die genannte Möglichkeit der Abschnürung in einem Divertikel geben, glaube aber nicht, dass das für unsere besondere Art ron Hydrocephalus zutrifft. Der Kopf müsste sonst erst das Divertikel verlassen haben, und sich dann zur Geburt eingestellt haben, denn eine Schädellage hat rorgelegen, wie auch die Kopfgeschwulst beweist. Ich 
muss also annehmen, dass der obere Kopiteil in der Uterushöhle selbst eine Einengung erfahren hat und dafür käme nur der untere wenig dehnungsfähige Teil der Uterushöhle Isthmus uteri in Betracht.

Wie gelangt nun der Kopf in so früher Zeit der. Gravidität in diesen engeren Teil des Uteruskanals? Dafür kann man zwei Möglichkeiten anführen; die eine wäre ein tiefer Eisitz, bei welchem, wie bekannt, zuerst ausschliesslich der untere Teil der Eihöhle dilatiert wird, die andere Möglichkeit wäre bei normalem Eisitz ein imperfekter Abort, bei welchem der Kopf in den isthmischen Uterusteil eingetrieben wird. Ob nun primär oder sekundär, das lässt sich nachträglich nicht entscheiden, jedenfalls scheint mir die Schädellage im starren, schlecht dilatierbaren unteren Uterusteile die beste Lösung dieser Frage. Nur der andauernde Widerstand einer wenig dehnungsfähigen Umgebung kann die Missbildung erklären und dazu scheint mir der untere Teil des Uterus am ehesten geeignet. - Es würde mich interessieren, das Urteil der Geburtshelfer hierüber zu erfahren.

Ich hatte Herrn Kollegen Dr. Zinnser gebeten, eine Nachuntersuchung nach dem Wochenbette der Frau vorzunehmen; er fand keine Abnormitäten am Uterus.

Den verhältnismässig ungewöhnlich grossen Schädeldefekt glaube ich mit der frühzeitigen Ausbildung des Hydrocephalus und mit den abnormen Druckverhältnissen (Einschnürung) in Zusammenhang. bringen zu müssen.

Ich glaubte, ihnen diesen Fall genauer vorführen zu dürfen, nicht nur wegen der genannten Schädelknochendefekte, sondern auch als sonst merkwürdige und besondere Form von Hydrocephalus internus, für die ich ein Seitenstück nur einmal in der Literatur. gefunden habe, nämlich bei Vrolick. (Tabulae ad illustrandam embryogenesin. Amsterdam 1849.) 